\title{
A knowledge-based view of analytics capability in purchasing and supply management
}

\author{
Mikael Öhman \\ Industrial Engineering and Management, Aalto University, Helsinki, Finland \\ Ala Arvidsson and Patrik Jonsson \\ Technology Management and Economics, Chalmers University of Technology, \\ Gothenburg, Sweden, and \\ Riikka Kaipia \\ Industrial Engineering and Management, Aalto University, Helsinki, Finland and \\ Technology Management and Economics, Chalmers University of Technology, \\ Gothenburg, Sweden
}

\begin{abstract}
Purpose - The purpose of this study is to elaborate on how analytics capability develops within the PSM function. This study is an in-depth exploration of how analytics capability develops within the purchasing and supply management (PSM) function.

Design/methodology/approach - A multiple case study was conducted of the PSM function of six case firms, in which primary data were collected through semi-structured interviews with PSM analytics stakeholders. The data were analyzed based on an analytics capability framework derived from the literature. Cases were chosen based on them having advanced PSM practices and ongoing analytics projects in the PSM area.

Findings - The findings shed light on how the firms develop their analytics capability in the PSM functional area. While we identify several commonalities in this respect, the authors also observe differences in how firms organize for analytics, bringing analytics and PSM decision-makers together. Building on the knowledge-based view of the firm, The authors offer a theoretical explanation of our observations, highlighting the user-driven side of analytics development, which has largely been unrecognized by prior literature. The authors also offer an explanation of the observed dual role that analytics takes in crossfunctional initiatives.

Research limitations/implications - The exploratory nature of our study limits the generalizability of our results. Further, our limited number of cases and interviewees indicate that there is still much to explore in the phenomenon of developing analytics capability.

Practical implications - Our findings can help firms gain a better understanding of how they could develop their analytics capability and what issues they need to consider when seeking leveraging data through analytics for PSM decisions.

Originality/value - This paper is, to the best knowledge of the authors, the first empirical study of analytics capability in PSM.
\end{abstract}

Keywords Data analytics, Analytics capability, Knowledge-based-view, Knowledge integration, Purchasing and supply management, Procurement

Paper type Research paper

(C) Mikael Öhman, Ala Arvidsson, Patrik Jonsson and Riikka Kaipia. Published by Emerald Publishing Limited. This article is published under the Creative Commons Attribution (CC BY 4.0) licence. Anyone may reproduce, distribute, translate and create derivative works of this article (for both commercial and non-commercial purposes), subject to full attribution to the original publication and authors. The full terms of this licence may be seen at http://creativecommons.org/licences/by/4.0/legalcode

The work was supported by the Chalmers Area of Advance Transport, in Sweden, and The Foundation for Economic Education (Liikesivistysrahasto) in Finland.

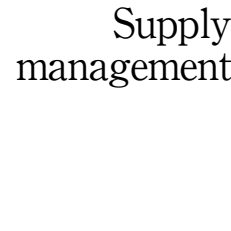

937

Received 17 December 2020 Revised 19 March 2021 24 June 2021

Accepted 9 July 2021 
IJPDLM

51,9

938

\section{Introduction}

Purchasing and supply management (PSM) as a functional area integrates the external environment and actors of the supply market with the internal stakeholders (Bals et al., 2019; Lorentz et al., 2020; Van Weele and Van Raaij, 2014). PSM decisions range from identification of potential external sources and suppliers and negotiation to operational management of suppliers, supply and spend (Van Weele and Van Raaij, 2014). In recent years, the explosion of data, access to such data and the potential of analytics have created the need to revisit which capabilities are necessary for the firm, and by extension, or even independently, its functions. We define analytics as all the activities that transform data into action, giving rise to new skills being needed, practices developed and governance principles established. The PSM function, specifically, requires capabilities to handle the large amounts of data accumulated internally, the supply market information (Lorentz et al., 2020; Schütz et al., 2020) and the integration of the generated insight with the relevant other functions internally at the firm (Arvidsson and Melander, 2020; Schütz et al., 2020) to satisfy both its cost-saving and long-term strategic purposes (Lorentz et al., 2020, 2021; Schütz et al., 2020; Van Weele and Van Raaij, 2014).

Leveraging analytics is a distinct and strategic capability (Davenport et al., 2001). The strategic role of PSM and the impact of PSM decisions on other functions and firm performance elevate the importance of analytics capability development in PSM. Previously, analytics related efforts focused on aspects of technology, but today the emphasis is on how data can contribute to business value through improving decision-making (Roßmann et al., 2018), impacting firm performance and competitiveness. This broadens the capability development challenge to include aspects such as governance, culture, and competencies (Mcafee and Brynjolfsson, 2012).

Reflecting general analytics related research, PSM research has been technology-focused, and hence only to a limited extent addressed the transformation of the function in the face of the new analytics challenges. Examples include discussion on contextual drivers and technological interventions in the digitalization of PSM (Lorentz et al., 2021) and the potential of certain technologies, such as big data analytics, cloud computing, automated procure-topay processes, real-time spend analytics, blockchain technology and virtual supplier rooms (Schmidt and Wagner, 2019; Srai and Lorentz, 2019). While Bals et al. (2019) note that technical skills related to analytics are increasingly in demand in PSM, it is safe to say that we still know little about the capability required to leverage analytics in PSM (Arvidsson $e$ al., 2021; Kosmol et al., 2019). In the fields of logistics and supply chain management, there are some recent research efforts toward understanding analytics capability (Herden, 2020).

Complementing prior technology-focused research on PSM analytics (Handfield et al., 2019; Lorentz et al., 2021; Srai and Lorentz, 2019) and firm-level analytics capability research (Roßmann et al., 2018), the purpose of this study is to elaborate on how analytics capability develops within the PSM function. The findings, thus, contribute not only to the understanding of analytics capability for the specific requirements of thePSM function butalso generate a more in-depth and detailed understanding of how analytics capability is manifested in the firm (cf. Zahra et al., 2020).

As analytics still promises considerable untapped potential, managers face an uncertain future, not knowing what analytics solutions will emerge, how they can be leveraged in business decision-making and what data are best used for which decision. In this highly uncertain situation, firms have to develop a strong set of capabilities that enable them to adapt their resources and processes to changing conditions and environments (Carrillo and Gaimon, 2004), not least within the PSM function (Lorentz et al., 2020). According to the knowledge-based view (KBV), firms build these capabilities through accessing and integrating specialized knowledge (Grant, 1996a, b; Nonaka, 1994). While figuring as the theoretical foundation of prior research in both analytics (Côrte-Real et al., 2017; Herden, 2020) and PSM (Carr and Pearson, 2002; Schütz et al.,2020), KBV-based research has been criticized for failing to elaborate on how firms actually integrate knowledge (Zahra et al., 2020). Based on our multiple case studies of PSM functions in six different firms, we seek to do exactly this, extending recent research in analytics (cf. Herden, 
2020) with our findings from the functional context of PSM. We also see a broader contribution in our findings on how the studied organizations facilitate cross-functional knowledge integration (Kotlarsky et al., 2015; Majchrzak et al., 2012; Zahra et al., 2020).

In the next section, we first develop an analytics capability frame of reference based on the KBV tenants and analytics literature - which guides our data collection and analysis. We then present our methodology for data collection and analysis, followed by findings, discussions on the findings and contributions of this paper.

\section{An analytics capability frame of reference}

\subsection{A knowledge-based view of the firm}

Building on the works of scholars such as Carrillo and Gaimon (2004), we understand knowledge to be both embedded in technical systems or physical capital resources and the human capital resources that are inherent within the firm's workforce. Knowledge includes the understanding of diverse scientific and engineering information, as well as specific skills necessary to effectively operate the technical systems. When integrated, knowledge creates value in a unique, inimitable and nontransferable way (Grant, 1996b). While knowledge is inherently created and held by individuals, as firm processes and routines get repeated, it gets embedded within the structures of the firm, moving in between the process of making individual knowledge available and connecting it to the firm's knowledge system (Grant, 1996b; Nonaka and von Krogh, 2009).

PSM knowledge, specifically, is related to both its traditional cost-saving and commercial role and also to its strategic role to have knowledge about supplier markets, external supplier environments, knowledge of their internal business partners and technical skills, as well as knowledge of the firm's overall strategic goals (Cousins et al., 2006). Schutz et al. (2020) find that the PSM knowledge and the proper application and configuration of such knowledge can drive firm performance. Consequently, in addition to individual-level knowledge, we can argue that knowledge is a highly relevant concept on both functional and firm levels (Zahra et al., 2020). Alongside actively developing knowledge, firms also need to integrate knowledge throughout the firm (Nonaka, 1994; Zahra et al., 2020), i.e. both within and across functions. This discussion is central to the position and role of the PSM function within the firm (Arvidsson and Melander, 2020; Schütz et al., 2020).

Individuals in the organization hold tacit knowledge, which is considered vital, as it is more difficult to imitate or transfer to competitors (held by specialists). However, tacit knowledge needs to be transferred and integrated internally within the function/firm (Grant, 1996b). Here, knowledge integration goes beyond the transfer of information (Fugate et al., 2012), having to overcome functional specialization (Kotlarsky et al., 2015) and difficulties in sharing that must be harmonized across the vested parties (Spekman and Davis, 2016). Herden (2020), studying knowledge in the context of analytics for logistics and supply-chain management suggests that integration of expertise in cross-functional teams is especially important e.g. in cross-functional sourcing situations.

Firm-level research on strategic IT decision-making suggests that strategic alignment within the organization requires integration of technological and business knowledge, implying shared domain knowledge between business managers and the technology (e.g. IT) managers (Ranganathan and Sethi, 2002). The shared knowledge is argued to improve communication and integration of planning processes (Kearns and Sabherwal, 2006). Contrasting these findings with that of Herden (2020), it is clear that analytics extends the responsibility for knowledge integration to include ordinary users, be they managers or not.

\subsection{Analytics capability as a competitive advantage}

As capabilities manifest in the complex interactions of a firm's resources, they are dependent on the firm's resources but cannot be acquired in the same way as resources (Teece and Leih, 2016). This principle also holds true for analytics capability, where having tools and management

939 
IJPDLM 51,9

940

technologies is necessary but an insufficient precondition to building competitive advantage on analytics (Acito and Khatri, 2014). In addition to (1) technology, analytics capability is also about (2) leadership or governance (Wang and Byrd, 2017), (3) culture (Akter et al., 2016), (4) methods (i.e. including people and processes) for turning data into knowledge (Erevelles et al., 2016) and integrating that knowledge within the firm (Côrte-Real et al., 2017). Similar to Cosic et al. (2015), these four aspects form the baseline for our conceptualization of analytics capability. Further, based on our review of analytics capability literature, we identified three cross-cutting themes, evidence-based decision-making, collaborative atmosphere and organizational adaptability, which we elaborate below, and which we cross-reference with the above four aspects, forming the basis for our analysis (see Appendix).

Analytics is the "evidence-based problem recognition and solving that happen[s] within the context of business situations" (Holsapple et al., 2014). Evidence-based management refers to firm culture and routine where managers base their decisions on facts and data (Pfeffer and Sutton, 2006). The PSM function has a historical tradition of managing large databases and making decisions based on facts. Having an evidence-based decision-making culture is seen as an integral part of analytics capability (Cosic et al., 2015; Vidgen et al., 2017). Pfeffer and Sutton (2006) contend that part of the problem for firms to become more evidence-based in their decision-making is the difficulty in validating and deciding how and for what to use the overwhelming amount of evidence from different sources; a struggle that we have also observed among the PSM managers that we interviewed. Further, Teece and Leih (2016) argue that strictly basing all managerial decisions on evidence can stifle decision-making. They argue that when an issue is at its early stages and has barely begun to unfold, the lack of evidence needs to be complemented by managerial insight based on expert knowledge. In PSM, a good example would be the sourcing of technology characterized by high uncertainty, where taking "a leap of faith" can be needed (Mikkelsen and Johnsen, 2019). Herden (2020) argues that an innovation promoting culture can be more important than being data-driven to gain a competitive advantage from analytics. With the PSM function's position and its role in capturing market innovation, such an argument also seems relevant in the context of our study.

Having a collaborative atmosphere in decision-making helps firms to access, shift and leverage market resources to rapidly respond to changes in a competitive environment. Firms that have a collaborative atmosphere should, therefore, be able to maintain high-performance levels over time (Fawcett et al., 2011). PSM ties both internally within the function and with other functions and their performance implications change over time and thus require a deeper analysis (Andersen and Gadde, 2019). Frisk and Bannister (2017) argue that in order to achieve performance improvements from analytics, managers need to be more collaborative in their decision-making processes. A collaborative decision-making atmosphere, openness and data sharing can assist the implementation of analytics (Sangari and Razmi, 2015). In this respect, firms need to balance between sharing and protecting (both intra- and inter-firm) data (Vidgen et al., 2017). The specific position and role of the PSM function within the firm puts PSM at the forefront of data sharing (Schütz et al., 2020). At the same time, the cost reduction objective of PSM can give rise to more data protection (Brattström and Richtnér, 2014).

Finally, through firm adaptability, firms can identify and respond to market threats and opportunities by quickly adjusting their actions. The concept is closely related to firm agility and operational flexibility (Chen et al., 2014). Teece et al. (2016) argue that firm agility relies mainly on management capable of combining and recombining technologies and malleable flexible structures. A rigid, hierarchical organizational structure has been associated with challenges in building an analytics capability (Côrte-Real et al., 2017). Yet, several studies assume that analytics development should be centralized, implying that a firm-level approach to analytics development is suitable (Fink et al., 2017; Vidgen et al., 2017). However, Fink (2017) notes that heterogeneous analytics needs within the firm imply that centralized development is not necessarily the best approach. 


\section{Methodology}

\subsection{Research design and case selection}

A multiple case study design was selected to study analytics capability development in PSM, as it allows rich and detailed data of the PSM analytics approaches in firms to be acquired (Yin, 2009). Case studies are suitable for studying contemporary phenomena within their context, with the multiple case study approach giving us a rich, in-depth view of the differences in analytics capability development in PSM. The unit of analysis is the analytics capability development within the context of PSM in a firm, in some cases being conducted by an analytics team within PSM, and in some cases by an analytics unit serving PSM decision-making in the case firm.

In seeking generalizable results (Yin, 2009), we used theoretical sampling, selecting firms that we considered advanced in their development efforts. Our sampling criteria included that (1) PSM should be of high priority, (2) there should be advanced supply management methods in use and (3) there should be an emphasis on and some advancement in PSM analytics. For access and convenience reasons, we chose case firms from Finland and Sweden, which matched our sampling criteria. Two of the case firms were identified in an industrial seminar about digitalization in purchasing. Two were identified among the respondents of a survey about analytics practices in PSM who had indicated a interest in a further study. Finally, two firms were identified based on earlier research collaboration, resulting in a total of six case firms. To ensure relevant, high-quality data, we had preliminary discussions with prospective cases to make sure that we would have access to key informants. Studying six cases allowed us to study diversified environments of analytics capability development, still obtaining deep into the analysis of each case based on rich data, as well as increasing external validity as comparative results could be analyzed through within-case analyses and a crosscase analysis, thereby utilizing a replication logic (Yin, 2009).

\subsection{Data collection}

Primary data was collected by interviewing key stakeholders in the case firms. Two rounds of data collection were conducted (see Table 1). In the first round, semi-structured interviews were conducted in each case firm. The contact people were selected based on the following criteria: they should be involved in PSM analytics, either be an analytics specialist, analytics manager or a person responsible for the firm unit dealing with analytics and PSM. When available, we selected more than one informant in order to allow triangulation and increase confidence in the results. The interviewees were identified with the help of contacts at the firms to ensure knowledgeable individuals on the topic. In the second round, the analysis and preliminary findings (i.e. their approaches to developing analytics) were presented to the representatives, their feedback gathered and an update on the analytics development made. All the management level interviewees (i.e. PSM managers, innovation managers and business intelligence managers) had been part of the purchasing department of their firms for more than 10 years, and the data analysts were recent hires (between one and five years) actively involved with the analytical projects today.

In each interview, two to four researchers were present, and interviewees were met individually or in pairs/groups (i.e. joint interviews). The interviews were conducted in English (cases A, B, F) and Finnish (cases C, D, E). Interviews were recorded and transcribed where possible and for those which were not recorded, at least two of the interviewers took detailed notes. The research team met after each interview to discuss the understanding from the interviews with the aim of avoiding misunderstandings and sharing insights. Other sources such as public documents were used to increase validity, as well as project reports from the earlier collaboration where available. Interviews were conducted face-to-face where possible or online.

A semi-structured interview protocol guided the discussion towards the objectives of the interview and gave the researchers the flexibility to focus on unique features in each case. The semi-structured interview guide was supported with a structured data collection guide to 


\section{IJPDLM \\ 51,9}

\section{2}

Table 1. Case overviews and sources of data
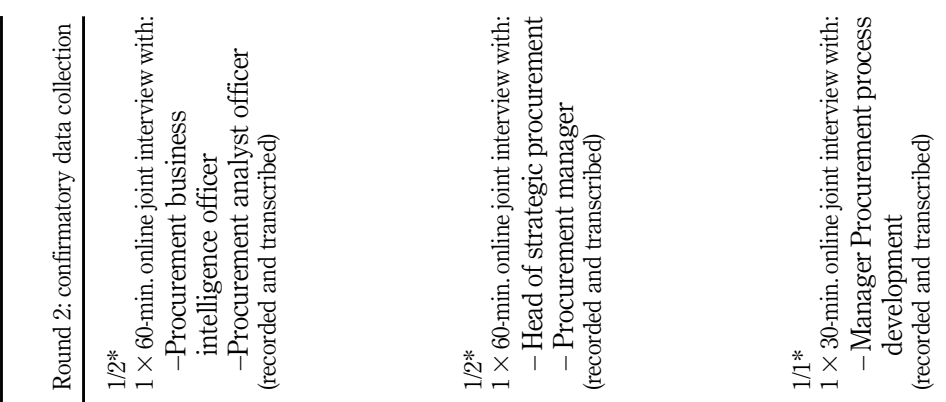

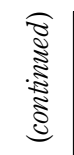

.

․

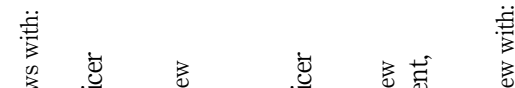

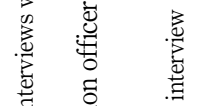

旁

表它总

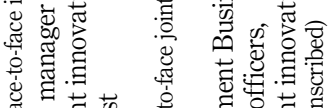

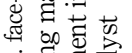

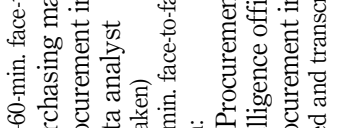

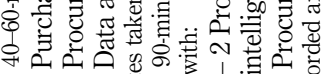

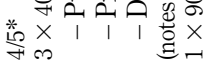

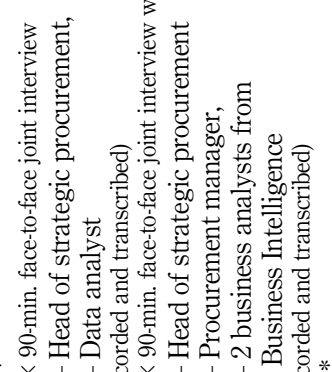

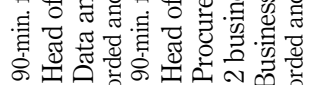

过旁 $\times$

苦蒙

.

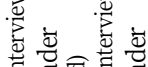
焉焉 可氜 ङ 设 记完要

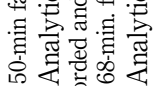
10
$\times 18$

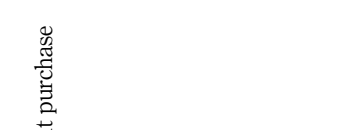

를

总. है:

올.

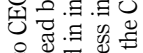

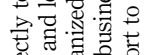

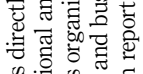

s.

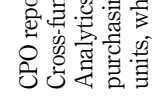
(

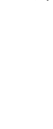
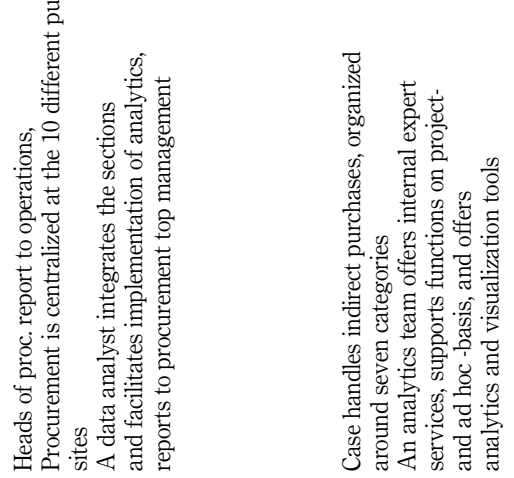

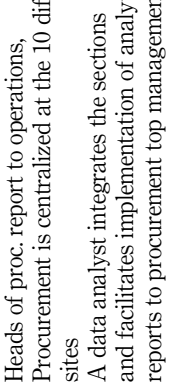

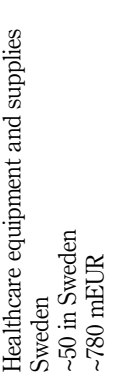

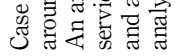

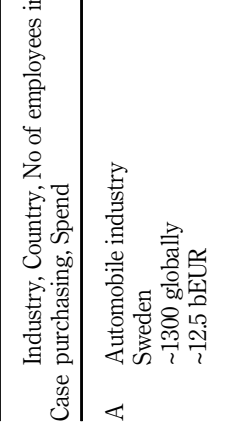

$\infty$

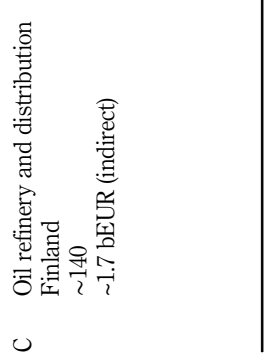




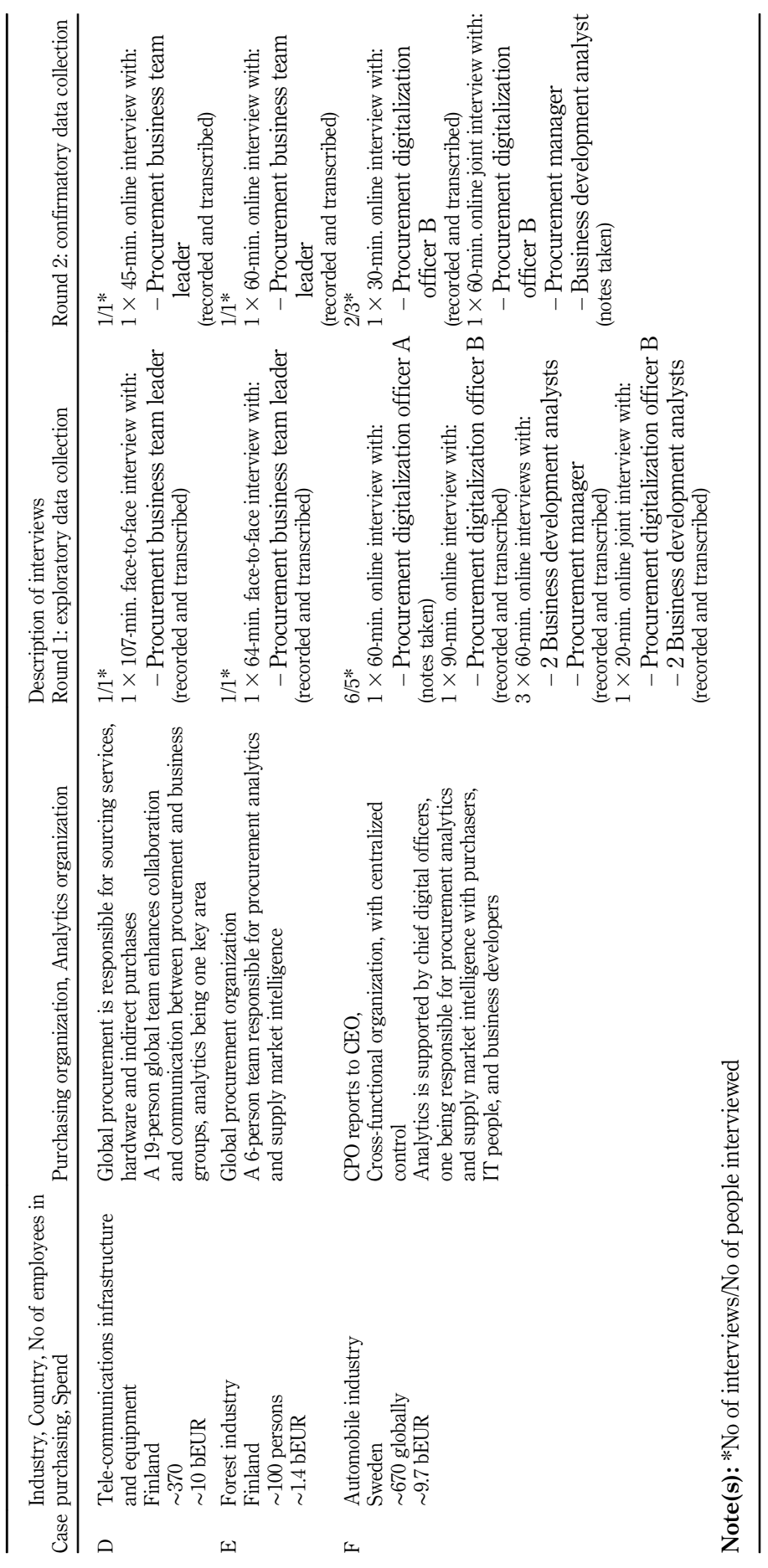

Supply

Table 1. 
IJPDLM 51,9

944

capturing the status of the analytics projects in the PSM area and the level of the capabilities. The interviews started with inquiries regarding the principles of PSM: ways of organizing, and main management priorities, after which the interviews proceeded to analytics in the PSM area. Here, questions were asked about the drivers and history of analytics, the decision-making culture of the firm, and examples of applications. Data management principles were discussed, along with the strategy for and organization of analytics. The relationship between analysts and decision-makers was studied, including the ways of communicating, how analytics related needs are identified and how analytics serves these needs. Lastly, performance and measurement, as well as insights about future development, were discussed.

\subsection{Data analysis}

The interview transcripts and notes were analyzed within cases and across cases (Miles and Huberman, 1994; Yin, 2009). The data analysis included phases with systematic coding, discussions among the researchers involved, write-ups of case descriptions, generation of different data displays, and visual projections of the coding results. Analysis and coding were done in English; the original interview data were translated by the researchers when needed, e.g. for quotations.

The transcribed interview data were coded with Atlas TI software, aiming to structure the data. Pre-defined codes for analytics capability, as identified in the literature identified above, were used, including the aspects of culture, governance, methods and technology, all of which were studied from the viewpoint of evidence-based decision-making, collaborative atmosphere and firm agility. Detailed descriptions of each of these 12 codes were written to ensure correct coding (see Appendix) and to create a common interpretation of each code. These capability-related codes were expected to cover the whole range of analytics capability development aspects. Importantly, code descriptions were allowed to be, and actually were, modified based on coded data from interviews, which were systematically listed for each code. Therefore, no new codes were added (see Appendix for coding principles).

The second group of pre-defined codes was created to describe the internal and external environment of the PSM function (see Appendix). Here, the purpose was to collect all quotes describing the contextual factors in each case, especially to work as potential contingencies, which may have an effect on how the analytics capability has developed and is developing. In this second code group, new codes were allowed to emerge - if a quote was identified as a potentially new contingency, the quote was assigned a new code.

This phase resulted in an overview of the analytics capability in each firm, which formed the basis for the cross-case analyses. At least two researchers participated in case coding and held several detailed discussions in order to ensure a similar interpretation of each quote and to extract data reductions about the pre-defined capability-related codes. The identified interpretations were discussed and modified between the researchers until consistency and consensus were reached. To complement within-case analysis, case descriptions were written with an emphasis on the context of the PSM function.

Next, projections of data extractions were created by synthesizing the findings and visualizing the approaches case firms have adopted in PSM analytics development. Here, the context factors were also connected to the approaches (see Appendix). Next, these observations were connected back to the tenants of the KBV, and based on this, the approaches the firms are using could be better understood, and differences and similarities across cases could be identified.

\subsection{Case descriptions}

All six case firms are manufacturing or refining firms. All firms have significant operations, such as manufacturing plants, in Sweden or Finland but are large multinational or global 
firms. Below, we briefly present the case firms, focusing on the analytics organization and its role in relation to the PSM function.

Case A is a large automotive manufacturer, and its advanced analytics for PSM is developed within innovation purchasing and business intelligence units. Both these units report directly to the $\mathrm{CPO}$. Various development ideas can be initiated anywhere in the purchasing organization, and if identified as a potential development solution, they get transferred to the innovation purchasing and/or business intelligence unit. The head of the innovation purchasing unit has the responsibility of identifying initiatives and promoting analytics solutions for PSM, among other innovation-related tasks such as joint projects with, e.g. research bodies. The business intelligence unit has the objective of increasing the share of and possibilities for purchasing decisions being made on data.

Case B is a manufacturer of healthcare supplies and equipment. The advanced analytics for PSM at the firm have been initiated by heads of two sections from amongst 10 PSM sections. There are no firm-level targets for PSM analytics, and development mainly stems from a personal drive. A data analyst has been hired to work across all the sections to facilitate the implementation of analytics in PSM, as well as the use of applications for supply risk management and spend management. The analyst reports to PSM top management, who report to operations management.

Case C is an oil refining firm's indirect purchases organized into seven categories: IT, logistics, technical services, travel and chemicals. The supply base is large and fragmented and varies from large global suppliers to local service providers. The analytics team was founded in 2014 as an "analytics and metrics" team. The team's role has developed from supporting competitive bidding, and development of data platforms, towards an internal expert service provider, which supports functions on project and ad hoc bases and offers analytics and visualization tools proactively to decision-makers.

Case D is a firm in the mobile technology industry, in which we focus on a global PSM business team of 19 people. The role of the team is to enhance the collaboration and communication between PSM and business groups in terms of service and support from PSM to businesses and expectations from businesses to PSM. In the firm, analytics is one of five key digital strategic initiatives. The firm can be described as data-driven and planningoriented, and it has a history of formal cross-firm processes to conduct operations planning.

Case $\mathrm{E}$ is a forest industry firm with PSM analytics development organized through a dedicated team, which has existed for ten years. The six-person team is responsible for PSM analytics and supply market intelligence, for collaboration with external analytics service providers, as well as systems and tools and the development of those. In PSM, analytics has evolved from spend-centric analytics to creating supply market intelligence. The goal of the team is to support purchasing strategy and to improve sourcing decisions.

Case $\mathrm{F}$ also operates in the automotive industry and has a dedicated digitalization officer who reports to the head of business development at the purchasing department. The firm has established a network of chief digitalization officers in each function. At the purchasing department, the chief digitalization officer works closely with the purchasers, IT people and business developers. The digitalization officer's tasks include educating the purchasing department, helping identify analytics opportunities, driving new analytics initiatives, and having an overview of ongoing and planned digital initiatives throughout the firm. The mission of the digitalization officer is to accelerate the digital transformation of purchasing at the firm, with a focus on analytics for direct material purchases; for example, in getting an overview of commercial relationships with suppliers and being able to predict their delivery performance.

\section{Findings}

In our cases, findings related to evidence-based decision-making and collaborative atmosphere overlapped to a large extent, especially in relation to the evidence of methods and practices in the 
IJPDLM 51,9

946

studied firms. For this reason, we will present the findings related to these two parts under the same heading. This intertwining nature of evidence-based decision-making and collaborative atmosphere is conveyed in the key finding below and further elaborated in Section 4.1:

Key finding 1: The studied PSM functions had distinct ways of bringing together data, analytics knowledge and PSM knowledge - comprising their analytics capability.

Further, it became apparent that the studied PSM functions at the different firms were in different phases of their effort to build their analytics capability, which was reflected in how the interviewees discussed the role of analytics and how analytics projects were prioritized.

We elaborate on the case-specific differences in Section 4.2, which support our second key finding:

Key finding 2: Analytics starts as a separate entity in the PSM function, needing to justify its existence while gradually becoming a part of daily life within the function.

\subsection{Evidence-based decision-making and collaborative atmosphere}

In order to develop an analytics capability, PSM decision-makers have to want to base their decisions on facts (versus intuition). All cases shared a history of an evidence-based decisionmaking culture. In case D, evidence-based decision-making is firmly rooted in their engineering industry, and "you would not last long if you made decisions based on gut feeling" (interviewee, case D). In case A, however, there is still a continued reliance on tacit knowledge in more complex PSM decisions.

I'd say that we use a lot of facts and data to support our decisions. We are a firm of engineers in that sense. Interviewee, case D

In cases $\mathrm{C}$ and $\mathrm{F}$, analytics played an active role in fostering and promoting evidence-based decision-making through various events, where cross-functional discussions were facilitated by analytics (case C), or where analytics' successes were showcased and promoted to spark wider interest in analytics (case F). Case D also saw that analytics is tasked with fostering an evidence-based decision-making culture, but in this case, the focus was not on encouraging the use of data (which was not a problem) but rather in making sure that decision-makers understand "what's behind the numbers" (interviewee, case D).

Finding 1.1: In all cases, the PSM function had a history of an evidence-based decisionmaking culture, and in some cases, analytics is actively shaping this culture.

All cases, quite naturally, saw data availability within the function as a key driver of analytics. In cases B, C and F, there was an expressed need to "bring the decision-maker closer to the data." However, the perceived impact of cross-functional data sharing varied between cases, with cases A, C and F considering it important. In cases $\mathrm{C}$ and $\mathrm{F}$ cross-functional data sharing was even seen as a driver of analytics development and the use of analytics. Yet, some data are more sensitive and could not be readily shared with the other functions in cases $\mathrm{A}$ and $\mathrm{F}$ (both from the automotive industry). Case $\mathrm{F}$ highlighted that openness with the other functions such as R\&D has to be accompanied by traceability.

So it's not like any employee can go and see any data that is available on the data lake. But still we want to make sure it's a thought-through case of why the data is needed and also to document to whom we've given access. Interviewee, Case $\mathrm{F}$

In case D, the analytics organization saw itself as the translator between PSM and the different product lines. Conversely, in case E, analytics focused on PSM data, as the width of categories were seen to reduce the impact of bringing in data from other functions. Case $\mathrm{E}$ also had competitive market relationships between some business lines, which put regulatory constraints on data sharing. Interestingly, although data quality was mentioned as a challenge in many cases, it was typically not seen as a major issue. 
Data quality has been one, but it may not be one of our biggest current challenges. Interviewee, Case E

Finding 1.2: Data availability in the PSM function was a key driver of analytics development; there were, however, differences in how important firm-wide data sharing was.

External data and analytics partnerships were leveraged to some extent in all cases, especially for the purpose of monitoring supplier and raw material markets. In cases A, B, C and $\mathrm{E}$ the (external) view of supply markets is something that is bought as a service, with case $\mathrm{F}$ also moving in this direction. Case $\mathrm{C}$ explicitly stated that some types of market knowledge were outside of the PSM analytics core competence and hence something to be bought as a service. Case $\mathrm{C}$ stressed that you need to have a good understanding of your analytics need before you can successfully outsource it.

We do not want to do everything, or even think that we can [...] there is always the expert somewhere out there who can do it for you. Interviewee, Case C

Related to analytics partnerships, Case E highlighted the need for centralized management to avoid the risk of costs getting out of hand. Although case D had several corporate-level groups dedicated to monitoring and analyzing the external business environment, they also used commercial market intelligence reports, which they discussed with suppliers to get further insights.

Finding 1.3: We found limited evidence of analytics solutions developed within the PSM function for monitoring the external business environment, as studied cases tended to use analytics service providers for this.

The PSM related knowledge of the analysts, or rather the lack thereof, was a common theme in all interviews, and in most cases, the challenge was expressed in terms of the analyst needing to gain a better understanding of the business (A, B, C, E and F). In cases C and $\mathrm{E}$, this was directly linked to a desire for analytics to be able to more proactively support decisionmakers, in contrast to a situation where analysts react to ill-expressed needs by the decisionmakers, resulting in unnecessary iterations and extra work for the analysts.

And development of analyst knowledge [...] so that analytics would be closer to the procurement process, [. . .], that analysts would have a better idea of, for example what categories do, [. . . and sort of technical stuff. So that he could proactively support better. Interviewee, Case $\mathrm{E}$

Cases $\mathrm{C}, \mathrm{E}$ and $\mathrm{F}$ also discussed measures taken by the analytics organization to increase the PSM decision-makers' knowledge of analytics. In cases $\mathrm{C}$ and $\mathrm{F}$, this was connected to a striving to increase decision-maker involvement in analytics work (which, in turn, was connected to a desire to make data available to decision-makers in the respective case). In case $\mathrm{D}$, the analytics team had an in-depth (business) understanding of both PSM and product lines, and it was the stated purpose of the analytics team to provide PSM with the relevant view of product lines and vice versa.

Finding 1.4: Most firms had room for improvement in how well analysts understood PSM decisions, but some also focused on improving PSM decision-makers' understanding of analytics.

In cases where analysts were being brought closer to the PSM decision-makers, taking part in the daily work of the function, there was also an expressed need for centralized support for analysts in terms of competence management and advanced analytics approaches $(\mathrm{C}$ and $\mathrm{F})$. This need was also recognized in case $\mathrm{E}$.

Finding 1.5: When analytics is done close to PSM decision-making, there is a need for centralized analytics competence management and (potentially) advanced analytics support.

Whereas in cases $\mathrm{A}$ and $\mathrm{E}$, the focus was clearly on developing analytics internally within $\mathrm{PSM}$, in cases $\mathrm{C}$ and $\mathrm{F}$ (PSM), analytics sought to actively create connections between 
IJPDLM

51,9

\section{8}

different parts of the business. This happens either through events intended for analysts (and business decision-makers) from different functions (cases $\mathrm{C}$ and $\mathrm{F}$ ), where success stories were presented (case F) or as direct facilitation of cross-functional opportunity spotting events which business decision-makers would attend (case C). Also, especially in case F, centralized analytics was organized in a way that fostered cross-functional opportunity recognition.

Case D was again special, as it comprised the link between the PSM and product lines; however, the non-hierarchical nature of the firm made collaboration easier (a point also brought up in case F). While case $\mathrm{E}$ focused on the internal development of PSM analytics, they noted that collaborating with highly independent local PSM functions was sometimes challenging.

Finding 1.6: In the cases where the PSM organization relied on cross-functional knowledge sharing, analytics actively sought to encourage and facilitate cross-functional collaboration.

\subsection{Organizational adaptability}

Given the gap between analytics and PSM knowledge, we found that analytics development projects and their prioritization contain a chicken-and-egg dilemma: do the resources for developing analytics come first or the business cases from which the firm gets its returns on invested resources. Related to this, we found an indication of a business case first -mentality in cases $\mathrm{A}$ and $\mathrm{E}$, which placed less emphasis on using analytics resources for exploration of potential business cases compared to cases, in which the interviewees did not feel they had constrained resources (cases $\mathrm{C}$ and $\mathrm{F}$ ). In line with their dual analytics development effort (finding 1.4), cases $\mathrm{C}$ and $\mathrm{F}$ seemed to follow more of an experimental approach (partly due to finding 2.2).

[...] It's more of a living organism. Maybe it's a not so rigid analytic roadmap going forward. It's more like we need to be encouraged to experiment. We are encouraged to try new tools to learn new skills. And focus on creating business value. Interviewee, Case F

Case $\mathrm{C}$ also related this to the distinction between reactive and proactive analytics. The former would be analysts working based on the needs and wants coming from the PSM decision-makers, and the latter would be analysts understanding the business needs well enough to be able to proactively create what the PSM decision-makers need or to provide them with data access and tools to create views from data, perhaps in a visual form.

Finding 2.1: Although all cases focused on creating business value, they differed in the extent to which the development of PSM analytics was driven by explicit business needs.

Finding 2.2: When analytics competences become more widespread at the firm, analytics focuses on making data available to the PSM decision-makers, which engages them in exploration based on their needs.

Cases $\mathrm{C}$ and $\mathrm{E}$ indicated moving from one mode of development to the other, however, in different directions. Case $\mathrm{C}$ stated that analytics work used to be reactive but is becoming more proactive (case C). Conversely, case $\mathrm{E}$ stated that they were moving in the other direction:

We are moving to a more focused development of analytics, as we have been in an exploration phase, where everyone is encouraged to experiment, and data are made available. Interviewee, Case $\mathrm{E}$

Related to the prior finding, we also observe that most interviewees noted that analytics need to justify itself in the PSM function, especially in the beginning (case A speaking in the present tense and cases $\mathrm{C}$ and $\mathrm{F}$ mainly speaking in the past tense) - translating to prioritization of a low-hanging fruit in analytics, which is relatively easy to implement and has considerable business impact. 
We have been picking the low-hanging fruit $[\ldots]$ and through that we have been able to create satisfaction and welcome this change. Interviewee, Case $\mathrm{C}$

Finding 2.3: As the PSM function begins to develop its analytics capability, the function's acceptance and justification for development efforts will be sought by focusing on solutions that are easy to implement and have notable business impact.

A number of other approaches were used to seek further acceptance within the function. These include the training of super-users (case B) or citizen data scientists (case F) within the PSM function, gradual introduction of analytics solutions through super-users (case B), limited testing communities (case A), user-centric design of analytics solutions (case A), and the high-profile promotion of analytics success stories (case F).

\section{Discussion}

Most prior research on analytics capability has seen it as a firm-level capability, whereas this study sought to investigate analytics capability at a functional level, within the PSM function. Reflected against prior analytics capability studies, our study provides insights into the considerable differences between how analytics capability is developed at a functional level. Building on KBV, developing analytics capability is essentially about how the firm manages to integrate analytics and PSM knowledge. We identified two distinct phases in how firms develop their analytics capability in PSM. Starting with a justification phase, where the focus is on intra-functional solutions, analytics knowledge and PSM knowledge is integrated, with analytics gradually being established as something that the firm does. As justification progresses in the PSM function (and other functions), the focus of analytics is increasingly on cross-functional initiatives. In cross-functional initiatives, analytics takes a dual role in the trifold integration of PSM, analytics and other functions' knowledge. We conclude the discussion by tying this duality to the KBV theoretical distinction between formal and tacit knowledge.

\subsection{Integration of analytics knowledge and PSM knowledge}

In the first phase, where analytics is new to the firm, the focus of analysts is to understand the PSM decision-maker needs. Spotting the opportunities in analytics is the responsibility of the individual(s) working with analytics. The more analytics is seen to constitute a change in how the firm works, the more it has to justify its existence and show its value. This justification is manifested in seeking quick victories, which shows business impact. Analytics provides a reactive service for the decision-maker, responding to ad-hoc requests by decision-makers. Successful development of analytics tools focuses on approachability and ease-of-use and acceptance of new solutions and analytics in general, spearheaded by "super-users" or the like. In our sample, cases A and B were positioned in this first phase, and in the validation discussions, they identified themselves as "gaining momentum" as per below.

The process of adoption entails analytics gaining momentum, as early analytics successes encourage the investment of more resources. Moving forward, the firm's analytics core competence and which analytics partnerships the firm should seek, both in terms of methods and data, become clearer (Herden, 2020). During this period, data become more accessible, and there are fewer and fewer problems with data quality. Shared knowledge between analysts and decision-makers grows, as an interaction between the two leads to the integration of analytics and PSM knowledge bases (see Figure 1).

In keeping with previous research, we found evidence-based decision-making to be the foundation of analytics capability (Cosic et al., 2015; Seddon et al., 2017). However, we also found that the methods for supporting evidence-based decision-making were intertwined with internal firm collaboration (Sangari and Razmi, 2015). Through the KBV lens, evidence-

management 
IJPDLM 51,9

\section{0}

Figure 1.

Analytics gains momentum in the justification phase

Figure 2.

Leveraging crossfunctional analytics opportunities through integration (left) and communication (right) based decision-making requires the overlap of analytics knowledge and PSM knowledge (e.g. knowledge of the exchanges and the supplier relationships, product knowledge, market knowledge and cross-functional engagement knowledge). In our cases, this was facilitated by different forms of collaboration between people with PSM knowledge and people with analytics knowledge. Facilitating this collaboration (Harvey, 2014) was one of the key activities that analytics was concerned with.

\subsection{Cross-functional knowledge integration through analytics}

In the second phase, as analytics within the function has become part of daily work, data are increasingly available within the firm; the focus of analytics development shifts toward crossfunctional opportunities (cf. Schütz et al., 2020). This happens through analytics taking two mutually supportive roles, depending on the extent of shared knowledge between PSM and the respective function. Based on how analytics positions itself in this tentative triad, these roles could be described as "integration" and "communication" (See Figure 2). The two roles should not be compared to each other in the sense that one would be more advanced than the other, but rather in terms of their suitability considering the context of PSM and the relationship between PSM and the other function.

In order for analytics to take an integration role, analytics knowledge has to be widespread in the PSM function and distinguishing between decision-makers and analysts should be difficult. Opportunity spotting, experimentation and even development are, to a large part,
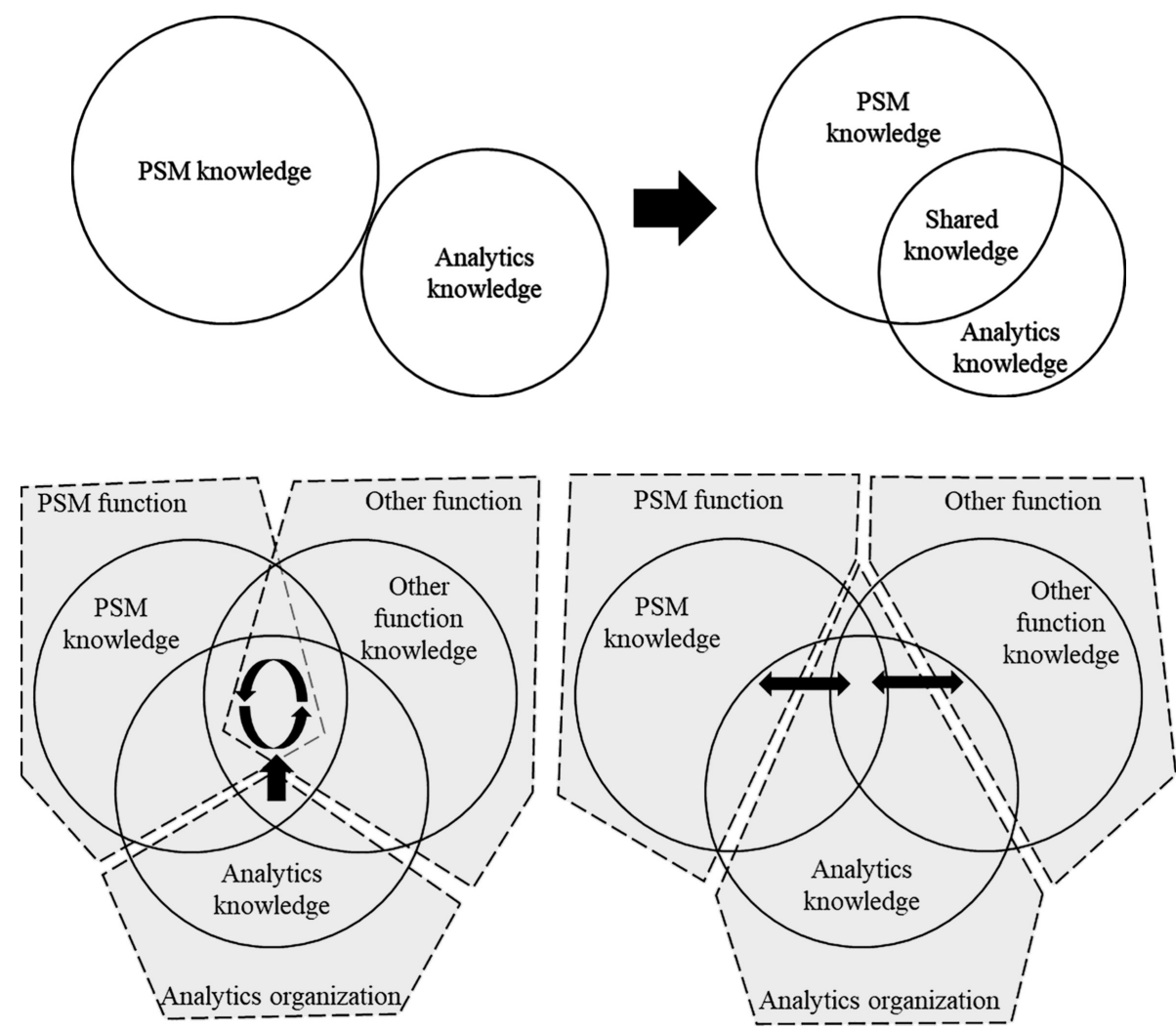
driven by analytics-savvy decision-makers who understand what data there are, where it can be found and what can be done with it. User-driven experimentation and development also mean that analytics shifts from prioritizing which projects should be prioritized to which projects should be disseminated. Analytics and centralized IT cooperate on making data available and useful to the decision-makers through unified data platforms or lakes and centralized data management. Analytics becomes a vocal advocate of evidence-based decision-making and arranges/facilitates events for cross-functional opportunity identification (cf. Harvey, 2014; Kotlarsky et al., 2015; Majchrzak et al., 2012), resulting in a trifold integration of PSM knowledge, analytics knowledge and other function knowledge, resulting, in turn, in the identification of new cross-functional analytics opportunities (Nonaka, 1994). We observed analytics taking an integrating role mainly in cases $\mathrm{C}$ and $\mathrm{F}$, where PSM worked in close collaboration with production.

In cases $\mathrm{D}$ and $\mathrm{E}$, where the (global/centralized) PSM function catered to several products, businesses and/or local organizations, we found that analytics took more of a communicating role, providing the firms with mutual insight into each other. In other words, instead of seeking to arrange/facilitate events for knowledge integration, analytics seeks to integrate the knowledge itself, acting as a gateway and interpreter between PSM and other internal organizations, mitigating knowledge boundaries (Kotlarsky et al., 2015). This role is manifested in analytics creating tools that provide a relevant view for PSM into different product lines and the business environment and vice versa. In terms of knowledge, the communication role requires an in-depth understanding of the business in the analytics team. Analytics still promotes an evidence-based culture, but the focus is more on educating decision-makers on what lies behind the numbers. In case $\mathrm{D}$, analytics also facilitated communication between PSM and suppliers through providing an innovation platform. The theoretical explanation would be that with less overlap of knowledge between PSM and the other function (compared to, e.g. cases $\mathrm{C}$ and $\mathrm{F}$ above), $\mathrm{PSM}$ is unable to spot cross-functional analytics opportunities (Nonaka, 1994) despite potentially having the required analytics knowledge to seize them (see also Kotlarsky et al., 2015). Further complications existed in case $\mathrm{E}$, where some business organizations were in a competitive relationship with each other, posing natural restrictions to knowledge integration.

Both KBV and capability-based research have been criticized for lack of detail with respect to processes, practices and organizing for knowledge integration (Zahra et al., 2020). The dual role of analytics in cross-functional initiatives (Kotlarsky et al., 2015; Majchrzak et al., 2012) adds detail in between the micro- and macro-levels of knowledge integration (Zahra et al., 2020). The trifold integration of PSM, analytics and other functions' knowledge was seemingly contingent on the redundancy (Nonaka, 1994) or overlap of functional knowledge bases (see also Kotlarsky et al., 2015), which we discuss next.

\subsection{On cross-functional integration of formal knowledge}

Considering the two different roles analytics takes in cross-functional initiatives, we posit that while codified data from another function might be easily accessible for decision-makers/ analysts in PSM, it still may fail to be informative for them. In other words, what is information, when viewed by someone at the other function (i.e. when understood using their tacit knowledge), may not be information for the PSM individual who might lack the relevant tacit knowledge.

From this follows an explanation of the theoretical conundrum, elaborating prior observations (Hildreth and Kimble, 2002; Nonaka and von Krogh, 2009) that formal knowledge is embedded with tacit knowledge from the realm in which it was created. The less redundancy there is in the knowledge base of the receiver of the formal knowledge and the knowledge base in which the formal knowledge originated, the less informative this formal 
IJPDLM 51,9

\section{2}

Figure 3.

How informative formal knowledge depends on whether the receiver has the relevant tacit knowledge (!) or not (?) knowledge is to the receiver (see Figure 3). Hence, the need for forums (cases $\mathrm{C}$ and $\mathrm{F}$ ) where tacit knowledge is integrated, simultaneously (returning to analytics research terminology for clarity) translating formal data into formal information.

This conceptualization was also supported by some of our other findings. An evidencebased decision-making culture necessitates decision-makers understanding what data the decision is based on - observed, especially in case D. Also, making sure that different internal organizations (e.g. business or product organizations) collect similar data in similar ways, leading to data becoming more understandable, i.e. informative, without the need for the tacit knowledge from the realm in which it was created - hence the drive to harmonize processes and data collection, which we recorded in the validation meeting with case $\mathrm{E}$.

Our observations of analytics capability development in PSM also tap into a persistent theoretical debate within KBV, namely on the nature of, and the relationship between formal and tacit knowledge (Hildreth and Kimble, 2002; Nonaka and von Krogh, 2009). Our findings, related to the knowledge integration role of analytics, highlight that a critical component of formal knowledge is the understanding of why that knowledge is relevant to the firm's stakeholders. While analytics research typically distinguishes between data and information (Delen and Demirkan, 2013), implying that data are not necessarily informative, KBV's distinction is mainly between tacit and formal knowledge. Considering how analytics change the way firms work with knowledge, we posit that KBV needs to integrate the former distinction as a tacit component of formal knowledge.

\section{Conclusions}

With this study, we aimed to answer how firms develop their PSM analytics capability using a KBV perspective. The differences we observed between our case firms indicate that there is no one-size-fits-all solution for how to develop analytics capability, supporting the criticism of firm-level analytics maturity models (Mccormack et al., 2009) and centrally driven analytics development (Fink et al., 2017).

Our contribution is first and foremost that we show that there are different paths in how analytics capability is developed within the firm while shedding much-needed light on the internal workings of analytics capability development in PSM (Lorentz et al., 2020; Schütz et al., 2020). Reflected against prior work, we identify a user-driven integration approach to analytics initiatives, highlighting opportunity-driven experimentation as a complement to the current problem-driven view of analytics development (Herden, 2020).

We advance the current understanding of how firms develop their analytics capability, by elaborating a duality in how knowledge is integrated in the PSM analytics capability (Zahra et al., 2020), extending previous analytics research based on the KBV (Herden, 2020). While analytics capability development in PSM starts with a justification phase where it gets

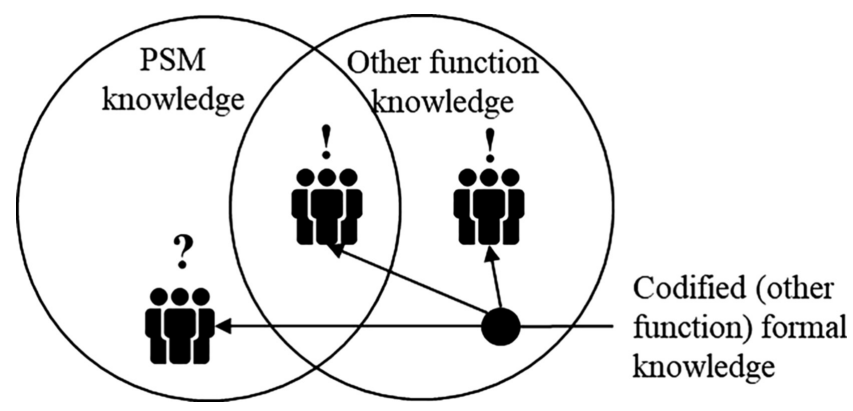


momentum and acceptance within the function, and which is in line with findings from other contexts (Herden, 2020), a dual role is identified afterwards. We found the dual role arising in response to cross-functional opportunities, with analytics acting as both an integrator and a communicator (cf. Kotlarsky et al., 2015), with the role being contingent on the redundancy (Nonaka, 1994), or the overlap of functional knowledge bases. Through these roles, we shed light on analytics as a mediator in cross-functional initiatives; as called for by Herden (2020).

\subsection{Practical implications}

The findings of this study have important implications for managers, both at the corporate and the functional level of the firms. At a functional level, the way data, analytics knowledge and PSM knowledge are brought together will shape PSM's analytics capability. At the corporate level, the study points out that linking analytics and business competences is especially crucial at the beginning of the analytics development effort, when analysts may have a quite shallow understanding of the business.

Making analytics accessible to decision-makers is crucial to analytics' acceptance and to the success of the integration pathway. Furthermore, making as much data available to the firm as possible seems to fuel analytics development. In this respect, it is important to consider the position of the PSM function within the firm, both in relation to the hierarchy and in relation to other functions, as the development of analytics capability seems to be tied to these firm structures.

For some, analytics is primarily about data and tools. While these are important, we wish to emphasize the cultural and collaborative aspects of analytics development. These aspects, along with an increasing understanding of the organizations' analytics core competences, can clarify many questions related to PSM. Including questions such as how analytics should advocate evidence-based decision-making within (an already data-driven) PSM, when to use external analytics solution providers, and how to strike a balance between local and centralized analytics development. As a tool for arriving at the right questions, we posit that our analysis framework (Appendix) could be useful for practitioners developing their analytics capability.

\subsection{Limitations and further research}

The exploratory nature of our study warrants careful interpretation of our findings. Although we found many similarities throughout the cases, we can, by no means, claim saturation of findings - indicating a need to conduct more exploratory research on PSM analytics capabilities in different firms and contexts. We argue that our choice of interviewees gave us broad and in-depth insight into the analytics capability development effort in the different firms. However, we do acknowledge that additional interviews would have provided a more nuanced view of the development process - indicating that getting more (especially decision-maker) perspectives of analytics capability development would probably generate exciting insights.

In our cases, there was a history of evidence-based decision-making at the PSM function, predating the systematic effort to build the PSM analytics capability, combined with a desire to be even more evidence-based. PSM is in this respect well positioned for developing an analytics capability, as there is a history of recording data and of data being available to their decision-makers. Also, perhaps due to this, data quality, which is often considered a major issue in analytics (Seddon et al., 2017; Vidgen et al., 2017), was not considered a significant challenge in the cases we studied. These observations point to PSM potentially being the most fruitful functional context for studying analytics capability development.

The capability development process, along with the complementary roles we present, requires further scrutiny. As we are dealing with two complementary ways of integrating management

953 
IJPDLM 51,9

knowledge (Zahra et al., 2020), there are several potentially fruitful theoretical vantage points for further inquiry, which could be combined with the notion of knowledge boundaries (Kotlarsky et al., 2015). Further research is required to gain a more fine-grained understanding of the complementary roles and the facilitating role of analytics (Harvey, 2014).

Further, we see that KBV can also provide a useful lens for understanding knowledge integration beyond the borders of the firm. Specifically in understanding outsourced analytics, both in cases of relying on external expertise for PSM knowledge (e.g. in-depth knowledge of raw-material markets, analytics related to which were outsourced in several of our cases) or methodological knowledge (e.g. in-depth methodological analytics knowledge, which was partially outsourced in some of our cases).

Finally, when analyzing case D, we also identified an underdeveloped area of research in how mergers and acquisitions shape the analytics capability.

\section{References}

Acito, F. and Khatri, V. (2014), "Business analytics: why now and what next?", Business Horizons, Vol. 57 No. 5, pp. 565-570.

Akter, S., Wamba, S.F., Gunasekaran, A., Dubey, R. and Childe, S.J. (2016), "How to improve firm performance using big data analytics capability and business strategy alignment?", International Journal of Production Economics, Vol. 182, pp. 113-131.

Andersen, P.H. and Gadde, L.E. (2019), "Organizational interfaces and innovation: the challenge of integrating supplier knowledge in LEGO systems", Journal of Purchasing and Supply Management, Vol. 25 No. 1, pp. 18-29.

Arvidsson, A.P. and Melander, L. (2020), "The multiple levels of trust when selecting suppliers insights from an automobile manufacturer", Industrial Marketing Management, Vol. 87, pp. 138-149.

Arvidsson, A.P., Jonsson, P. and Kaipia, R. (2021), "Big data in purchasing and supply management: a research agenda”, International Journal of Procurement Management, Vol. 14 No. 2, pp. 185-212.

Bals, L., Schulze, H., Kelly, S. and Stek, K. (2019), "Purchasing and supply management (PSM) competencies: current and future requirements", Journal of Purchasing and Supply Management, Vol. 25 No. 5, 100572.

Brattström, A. and Richtnér, A. (2014), "Good cop-bad cop: trust, control, and the lure of integration”, Journal of Product Innovation Management, Vol. 31 No. 3, pp. 584-598.

Carr, A.S. and Pearson, J.N. (2002), "The impact of purchasing and supplier involvement on strategic purchasing and its impact on firm's performance", International Journal of Operations and Production Management, Vol. 22 No. 9, pp. 1032-1053.

Carrillo, J.E. and Gaimon, C. (2004), "Managing knowledge-based resource capabilities under uncertainty", Management Science, Vol. 50 No. 11, pp. 1504-1518.

Chen, Y., Wang, Y., Nevo, S., Jin, J., Wang, L. and Chow, W.S. (2014), "IT capability and organizational performance: the roles of business process agility and environmental factors", European Journal of Information Systems, Vol. 23 No. 3, pp. 326-342.

Côrte-Real, N., Oliveira, T. and Ruivo, P. (2017), "Assessing business value of big data analytics in European firms", Journal of Business Research, Vol. 70, pp. 379-390.

Cosic, R., Shanks, G. and Maynard, S. (2015), “A business analytics capability framework", Australasian Journal of Information Systems, Vol. 19, pp. S5-S19.

Cousins, P.D., Lawson, B. and Squire, B. (2006), "An empirical taxonomy of purchasing functions", International Journal of Operations and Production Management, Vol. 26 No. 7, pp. 775-794.

Davenport, T.H., Harris, J.G., De Long, D.W. and Jacobson, A.L. (2001), "Data to knowledge to results: building an analytic capability", California Management Review, Vol. 43 No. 2, pp. 117-138. 
Delen, D. and Demirkan, H. (2013), "Data, information and analytics as services", Decision Support Systems, Vol. 55 No. 1, pp. 359-363.

Erevelles, S., Fukawa, N. and Swayne, L. (2016), "Big data consumer analytics and the transformation of marketing", Journal of Business Research, Vol. 69 No. 2, pp. 897-904.

Fawcett, S.E., Wallin, C., Allred, C., Fawcett, A.M. and Magnan, G.M. (2011), "Information technology as an enabler of supply chain collaboration: a dynamic-capabilities perspective", Journal of Supply Chain Management, Vol. 47 No. 1, pp. 38-59.

Fink, L., Yogev, N. and Even, A. (2017), "Business intelligence and organizational learning: an empirical investigation of value creation processes", Information and Management, Vol. 54 No. 1, pp. 38-56.

Frisk, J.E. and Bannister, F. (2017), "Improving the use of analytics and big data by changing the decision-making culture: a design approach", Management Decision, Vol. 55 No. 10, pp. 2074-2088.

Fugate, B., Autry, C. and Davis-Sramek, B. (2012), "Does knowledge management facilitate logisticsbased differentiation? The effect of global manufacturing reach", International Journal of Production Economics, Vol. 139 No. 2, pp. 496-509.

Grant, R.M. (1996a), "Prospering in dynamically-competitive environments: organizational capability as knowledge integration", Organization Science, Vol. 7 No. 4, pp. 375-387.

Grant, R.M. (1996b), "Toward a knowledge-based theory of the firm”, Strategic Management Journal, Vol. 17 No. S2, pp. 109-122.

Handfield, R., Jeong, S. and Choi, T. (2019), "Emerging procurement technology: data analytics and cognitive analytics", International Journal of Physical Distribution and Logistics Management, Vol. 49 No. 10, pp. 972-1002.

Harvey, S. (2014), "Creative synthesis: exploring the process of extraordinary groups creativity", The Academy of Management Review, Vol. 39 No. 3, pp. 324-343.

Herden, T.T. (2020), "Explaining the competitive advantage generated from analytics with the knowledge-based view: the example of logistics and supply chain management", Business Research, Vol. 13 No. 1, pp. 163-214.

Hildreth, P.M. and Kimble, C. (2002), "The duality of knowledge", Information Research, Vol. 8 No. 1, pp. 1-18.

Holsapple, C., Lee-Post, A. and Pakath, R. (2014), "A unified foundation for business analytics", Decision Support Systems, Vol. 64, pp. 130-141.

Kearns, G.S. and Sabherwal, R. (2006), "Strategic alignment between business and information technology: a knowledge-based view of behaviors, outcome, and consequences", Journal of Management Information Systems, Vol. 23 No. 3, pp. 129-162.

Kosmol, T., Reimann, F. and Kaufmann, L. (2019), "You'll never walk alone: why we need a supply chain practice view on digital procurement", Journal of Purchasing and Supply Management, Vol. 25 No. 4, 100553.

Kotlarsky, J., van den Hooff, B. and Houtman, L. (2015), "Are we on the same page? Knowledge boundaries and transactive memory system development in cross-functional teams", Communication Research, Vol. 42 No. 3, pp. 319-344.

Lorentz, H., Aminoff, A., Kaipia, R. and Srai, J.S. (2021), "Structuring the phenomenon of procurement digitalisation: contexts, interventions and mechanisms", International Journal of Operations and Production Management, Vol. 41 No. 2, pp. 157-192.

Lorentz, H., Aminoff, A., Kaipia, R., Pihlajamaa, M., Ehtamo, J. and Tanskanen, K. (2020), “Acquisition of supply market intelligence - an information processing perspective", Journal of Purchasing and Supply Management, Vol. 26 No. 5, 100649.

Majchrzak, A., More, P.H.B. and Faraj, S. (2012), "Transcending knowledge differences in crossfunctional teams", Organization Science, Vol. 23 No. 4, pp. 951-970.

\section{management}

Supply 
IJPDLM 51,9

Mcafee, A. and Brynjolfsson, E. (2012), "Big data: the management revolution”, Harvard Business Review, Vol. 90 No. 10, pp. 60-68.

Mccormack, K., Willems, J., Van Den Bergh, J., Deschoolmeester, D., Willaert, P., Štemberger, M.I., Škrinjar, R., Trkman, P., Ladeira, M.B., de Oliveira, M.P.V., Vuksic, V.B. and Vlahovi, N. (2009), "A global investigation of key turning points in business process maturity", Business Process Management Journal, Vol. 15 No. 5, pp. 792-815.

Mikkelsen, O.S. and Johnsen, T.E. (2019), "Purchasing involvement in technologically uncertain new product development projects: challenges and implications", Journal of Purchasing and Supply Management, Vol. 25 No. 3, 100496.

Miles, M.B. and Huberman, A.M. (1994), Qualitative Data Analysis: an Expanded Sourcebook, 2nd ed., Sage Publications, Thousand Oaks, CA.

Nonaka, I. (1994), "A dynamic theory of organizational knowledge creation”, Organization Science, Vol. 5 No. 1, pp. 14-37.

Nonaka, I. and von Krogh, G. (2009), "Tacit knowledge and knowledge conversion: controversy and advancement in organizational knowledge creation theory", Organization Science, Vol. 20 No. 3, pp. 635-652.

Pfeffer, J. and Sutton, R.I. (2006), "Evidence-based management”, Harvard Business Review, Vol. 84 No. 1, pp. 62-74.

Ranganathan, C. and Sethi, V. (2002), "Rationality in strategic information technology decisions: the impact of shared domain knowledge and IT unit structure", Decision Sciences, Vol. 33 No. 1, pp. 59-86.

Roßmann, B., Canzaniello, A., von der Gracht, H. and Hartmann, E. (2018), "The future and social impact of big data analytics in supply chain management: results from a Delphi study", Technological Forecasting and Social Change, Vol. 130, pp. 135-149.

Sangari, M.S. and Razmi, J. (2015), "Business intelligence competence, agile capabilities, and agile performance in supply chain: an empirical study", International Journal of Logistics Management, Vol. 26 No. 2, pp. 356-380.

Schmidt, C.G. and Wagner, S.M. (2019), "Blockchain and supply chain relations: a transaction cost theory perspective", Journal of Purchasing and Supply Management, Vol. 25 No. 4, 100552.

Schütz, K., Kässer, M., Blome, C. and Foerstl, K. (2020), "How to achieve cost savings and strategic performance in purchasing simultaneously: a knowledge-based view", Journal of Purchasing and Supply Management, Vol. 26 No. 2, 100534.

Seddon, P.B., Constantinidis, D., Tamm, T. and Dod, H. (2017), "How does business analytics contribute to business value?”, Information Systems Journal, Vol. 27 No. 3, pp. 237-269.

Spekman, R. and Davis, E.W. (2016), "The extended enterprise: a decade later", International Journal of Physical Distribution and Logistics Management, Vol. 46 No. 1, pp. 43-61.

Srai, J.S. and Lorentz, H. (2019), "Developing design principles for the digitalisation of purchasing and supply management", Journal of Purchasing and Supply Management, Vol. 25 No. 1, pp. 78-98.

Teece, D. and Leih, S. (2016), "Uncertainty, innovation, and dynamic capabilities: an introduction", California Management Review, Vol. 58 No. 4, pp. 5-12.

Teece, D., Peteraf, M. and Leih, S. (2016), "Dynamic capabilities and organizational agility: risk, uncertainty, and strategy in the innovation economy", California Management Review, University of California Press, Vol. 58 No. 4, pp. 13-35.

Van Weele, A.J. and Van Raaij, E.M. (2014), "The future of purchasing and supply management research: about relevance and rigor", Journal of Supply Chain Management, Vol. 50 No. 1, pp. 56-72.

Vidgen, R., Shaw, S. and Grant, D.B. (2017), "Management challenges in creating value from business analytics”, European Journal of Operational Research, Vol. 261 No. 2, pp. 626-639. 
Wang, Y. and Byrd, T.A. (2017), "Business analytics-enabled decision-making effectiveness through knowledge absorptive capacity in health care", Journal of Knowledge Management, Vol. 21 No. 3, pp. 517-539.

Yin, R.K. (2009), Case Study Research: Design and Methods, 4th ed., Sage Publications Ltd., Thousand Oaks, CA.

Zahra, S.A., Neubaum, D.O. and Hayton, J. (2020), "What do we know about knowledge integration: fusing micro-and macro-organizational perspectives", Academy of Management Annals, Vol. 14 No. 1, pp. 160-194.

\section{Appendix}

The Appendix file is available online for this article.

\section{Corresponding author}

Ala Arvidsson can be contacted at: ala.arvidsson@chalmers.se

For instructions on how to order reprints of this article, please visit our website:

www.emeraldgrouppublishing.com/licensing/reprints.htm

Or contact us for further details: permissions@emeraldinsight.com 